\title{
Nanoscale
}

Check for updates

Cite this: Nanoscale, 2019, 11, 2848

\section{Three dimensional nanoscale analysis reveals aperiodic mesopores in a covalent organic framework and conjugated microporous polymer $\dagger$}

\author{
Craig T. Stoppiello, (D) a Helena Isla, ${ }^{b}$ Marta Martínez-Abadía, (DD ${ }^{b}$ Michael W. Fay, (DD ${ }^{c}$ \\ Christopher D. J. Parmenter, ${ }^{c}$ Martin J. Roe, ${ }^{c}$ Belén Lerma-Berlanga, ${ }^{d}$ \\ Carlos Martí-Gastaldo, (D) d Aurelio Mateo-Alonso (iD ${ }^{\text {b,e }}$ and \\ Andrei N. Khlobystov (iD) *a,c
}

Received 21st December 2018 , Accepted 4th January 2019

DOI: $10.1039 / \mathrm{c} 8 \mathrm{nr} 10086 \mathrm{a}$

rsc.li/nanoscale

\begin{abstract}
The integrated analytical approach developed in this study offers a powerful methodology for the structural characterisation of complex molecular nanomaterials. Structures of a covalent organic framework based on boronate esters (COF-5) and a conjugated microporous polymer (Aza-CMP) have been investigated by a combination of several electron microscopy techniques elucidating the three-dimensional topology of the complex polycrystalline (COF) and non-crystalline (CMP) materials. Unexpected, aperiodic mesoporous channels of 20-50 nm in diameter were found to be penetrating the COF and CMP particles, which cannot be detected by X-ray diffraction techniques. The mesopores appear to be stable under a range of different conditions and accessible to gas molecules, exhibiting a particular bonding capability with $\mathrm{CO}_{2}$ in the case of the CMP. The mesoporosity is unrelated to the intrinsic chemical structures of the COF or CMP but rather it reflects the mechanisms of polymer particle formation in a polycondensation reaction. The mesopores may be templated by clusters of solvent molecules during the COF or CMP synthesis, leaving cavities within the polymer particles. The unexpected mesoporosity discovered in COF and CMP materials begs for re-assessment of the nature of framework materials and may open new opportunities for applications of these molecular materials in gas sorption or catalysis.
\end{abstract}

\section{Introduction}

The structural characterisation of low-crystalline, disordered or aperiodic solids remains one of the biggest challenges faced by the chemistry community, ${ }^{1}$ but it is vitally important for harnessing the properties of many enigmatic disordered molecular materials. ${ }^{2}$ New types of porous materials, such as covalent organic frameworks (COFs) ${ }^{3}$ and conjugated microporous polymers (CMPs) ${ }^{4}$ attract much interest as synthetic organic 2D materials, that offer many functional properties that could be exploited for uses in gas storage, ${ }^{5}$ sensing, ${ }^{6,7}$ drug delivery ${ }^{8,9}$ or

\footnotetext{
${ }^{a}$ The School of Chemistry, University of Nottingham, Nottingham, NG7 2RD, UK. E-mail: Andrei.Khlobystov@nottingham.ac.uk

${ }^{b}$ POLYMAT, University of the Basque Country UPV/EHU, Avenida de Tolosa 72 , 20018 Donostia-San Sebastian, Spain

${ }^{c}$ The Nanoscale and Microscale Research Centre, University of Nottingham, Nottingham, NG7 2RD, UK

${ }^{d}$ Instituto de Ciencia Molecular Universitat de València, Catedrático José Beltrán 2, 46980 Paterna, Spain

${ }^{e}$ Ikerbasque, Basque Foundation for Science, Bilbao, Spain

$\dagger$ Electronic supplementary information (ESI) available. See DOI: 10.1039/ c8nr10086a
}

catalysis, ${ }^{10,11}$ in addition to a microporous structure allowing for the tuning of their electronic bandgap. ${ }^{12,13}$ However, the complex and often disordered 3D structures of CMPs and COFs to some extent present a significant hurdle for establishing structure-property relations and thus hinder the development of practical applications for these innovative materials. ${ }^{14}$

Among the structural characterisation methods, threedimensional electron diffraction tomography has previously been effectively utilised to probe the internal structure of flat ordered COF particles. ${ }^{15,16}$ However, diffraction requires a high degree of crystallinity which often is not possible in their less crystalline analogues, such as CMPs. In addition, any aperiodic structural features or defects in the range of $20 \mathrm{~nm}$ or above will be simply invisible for the diffraction techniques, electron beam or X-ray alike. In contrast, direct space imaging by transmission electron microscopy (TEM) can be successfully used for non-crystalline materials and has been shown to provide valuable information for highly ordered COFs but only limited information for less ordered CMP particles. ${ }^{8,17-21}$

Herein we demonstrate a methodology that can be effectively utilised for the structural analysis of both a crystalline COF and a highly disordered CMP, relying on a complementary 
combination of focussed ion beam scanning electron microscopy (FIB-SEM) and TEM tomography that allows for the detailed evaluation of the complex structures across the length scales and reveals mesoporous channels which originate not from the intrinsic chemical structures of either the COF or CMP but from the mechanism of the polymer particle growth. For this, two previously reported COFs and CMPs have been examined. The boronate ester $\mathrm{COF}-5^{22}$ and the more disordered Aza-CMP, ${ }^{23-25}$ are examples of materials where the size of the micropores can greatly influence the electronic properties $^{25-27}$ but establishing the structure of such materials is extremely difficult.

\section{Results and discussion}

High-resolution (HR) TEM imaging of the COF-5 particles deposited on amorphous carbon film on a copper TEM grid allows clear visualisation of the overall shape of a particle which appears as roughly rectangular (Fig. 1a and d). Energy dispersive X-ray (EDX) analysis confirms the presence of all elements; carbon, oxygen and boron (with the latter overlapping with the carbon peak), expected from the atomic composition of the COF-5 (Fig. 1b), with electron energy loss spectroscopy (EELs Fig. 1c) confirming the presence of boron within the material. Areas of the particle hanging over a hole in the carbon film imaged at higher magnification clearly reveal linear channels with diameters of 3-3.5 nm corresponding to projections of highly ordered hexagonal pores of this material that give rise to sharp peaks in the powder XRD pattern (ESI, Fig. S1 $\dagger$ ). The sets of parallel linear channels run continuously through domains of only $\sim 50 \mathrm{~nm}$ rather than the entire COF particle indicating a polycrystalline nature (Fig. 1a, inset). It is important to note that the COF is very susceptible to the electron beam damage as after only a modest irradiation of the COF by a $200 \mathrm{keV}$ e-beam up to a dose of $\sim 10^{8} \mathrm{e} \mathrm{nm}^{2}$ the channels begin collapsing due to the onset of amorphisation caused by the e-beam that destroys the nanoscale structure. However, the overall particle morphology and geometry, and the features in the range of $10 \mathrm{~nm}$ or above in the COF remain effectively unchanged even after very high doses of irradiation. a)

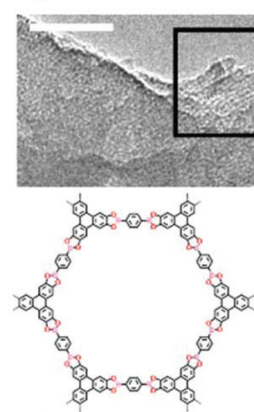

d)



b)

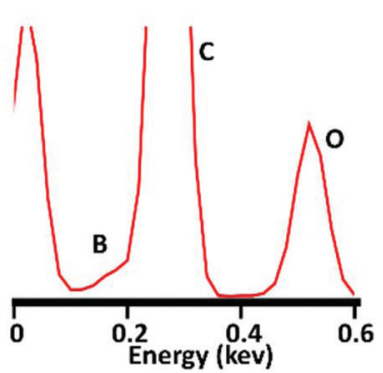

c)

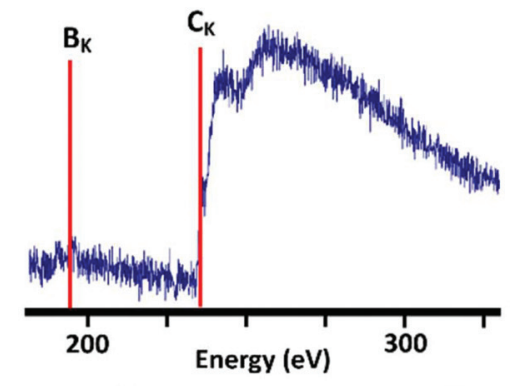

e)
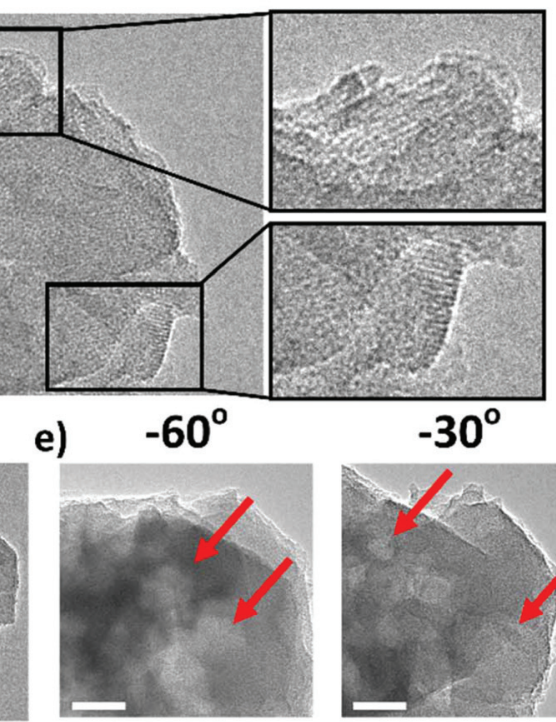

$-30^{\circ}$


$0^{\circ}$

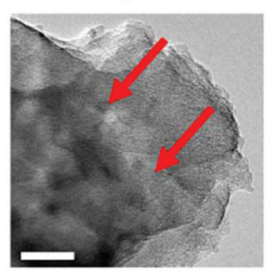

$+30^{\circ}$

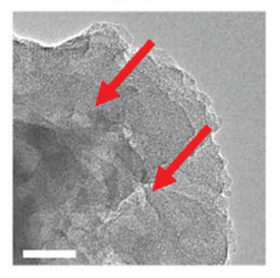

$+60^{\circ}$

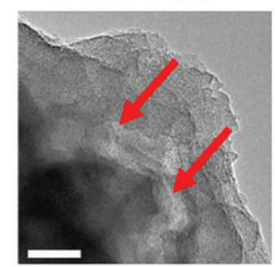

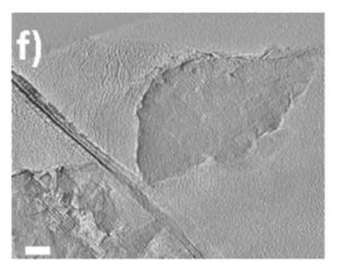
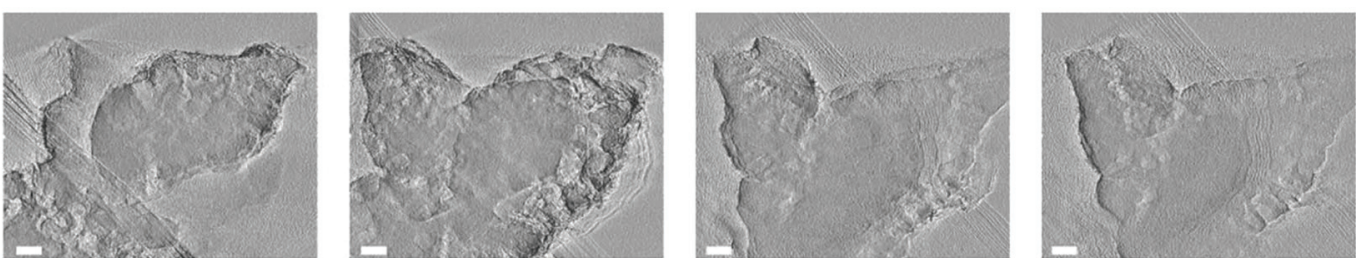

Fig. 1 (a) A bright-field HR-TEM image of COF-5 (structural diagram is shown in the bottom left corner) demonstrating the crystalline nature of the particle and the presence of linear channels which are associated with the hexagonal pores in a side-on projection (inset) the scale bar is $50 \mathrm{~nm}$; (b) an EDX spectrum taken over the particle in (a) showing C, O, and B present; (c) EELs shows B and C within the sample, (d) overall view (low magnification) of the COF-5 particle showing a non-uniform contrast associated with different thicknesses, the scale bar is $100 \mathrm{~nm}$; (e) TEM tomographic tilt series showing the COF-5 particle tilted through $120^{\circ}$ allowing a comprehensive visualisation in three dimensions of the mesoporous structure (red arrows indicate some prominent mesopores), the scale bars are $50 \mathrm{~nm}$; (f) varying z-slices from the SIRT of COF-5 showing mesopores within the particle, the scale bars are $50 \mathrm{~nm}$. 
The large field of view images of the COF- 5 particle demonstrates a non-uniform, 'speckled' overall particle contrast with the areas of high crystallinity ( $\sim 50 \mathrm{~nm}$, darker regions) interconnected with lighter regions of 10-50 $\mathrm{nm}$ which can be interpreted as voids (Fig. 1d). Since a single TEM image demonstrates a two-dimensional projection of a three-dimensional object, it is insufficient to provide detailed microstructural analysis, but it can be concluded that the 'speckled' contrast of the TEM image is due to the thickness contrast mechanism. Thus, assuming that each TEM image is effectively a twodimensional model of the attenuation coefficient through the sample, it is possible to obtain a tilt-series (Fig. 1e series, ESI Video $\mathrm{V} 1 \dagger$ ) and process the data to extract three-dimensional information. ${ }^{28}$ The use of a simultaneous iterative reconstruction technique (SIRT) has given a complex model showing how the contrast alters as a function of position within the particle, and effectively enables us to observe aperiodic mesopores within the COF-5 particle, appearing as areas of lighter contrast, with sizes of tens of $\mathrm{nm}$ penetrating the entire particle (Fig. 1f series, ESI Video V2 $\dagger$ ). Therefore, the COF-5 material can be described as nanocrystalline, with clearly defined nanoporous and mesoporous structures coexisting within the same particle.

Next, this approach was extended to the structurally more challenging Aza-CMP as an example of a highly disordered framework material. ${ }^{23-25}$ Indeed, bright-field TEM imaging of the Aza-CMP (Fig. 2a) shows discrete flat particles with lateral dimensions of around $100 \mathrm{~nm}$ and no periodic features
(Fig. 2b) as in COF-5. Inhomogeneous contrast of the material suggests that the extended covalent network is a layered twodimensional structure of varied thickness, and HR-TEM imaging shows the edge of one of the terrace-like structures in more detail, suggesting that Aza-CMP is intrinsically non-crystalline (Fig. 2c, ESI Fig. S5 $\dagger$ ). The presence of carbon and nitrogen in the structure is confirmed by EELs (Fig. 2d), and both elemental mapping (Fig. 2e) and EDX (Fig. 2f) further show the occurrence of carbon and nitrogen consistent with the chemical composition of Aza-CMP, but also indicate that traces of oxygen are present, which is unexpected given the chemical composition of the material (Fig. 2a).

Similarly to COF-5, a large field of view of the Aza-CMP shows a non-uniform contrast variation. A tilt-series for AzaCMP (Fig. 3a series, ESI Video V3†) reveals mesopores within the particle, appearing as areas of lighter contrast, and a SIRT elaborates the complex 3D morphology of the channels with sizes of tens of $\mathrm{nm}$ (Fig. 3b series, ESI Videos V4-V6†). The aza-CMP material appears to be much more stable in the e-beam than COF-5 with no observable changes in its structure or morphology even after extensive doses of the e-beam which enabled the acquisition of maps of element distribution (Fig. 2e). Furthermore, this unexpected stability allowed us to apply a focussed ion beam (FIB) to etch the surface layer of the particles and directly reveal the sub-surface of the Aza-CMP structure. Used in conjunction with scanning electron microscopy (SEM) we exposed the CMP to a beam of $30 \mathrm{keV}$
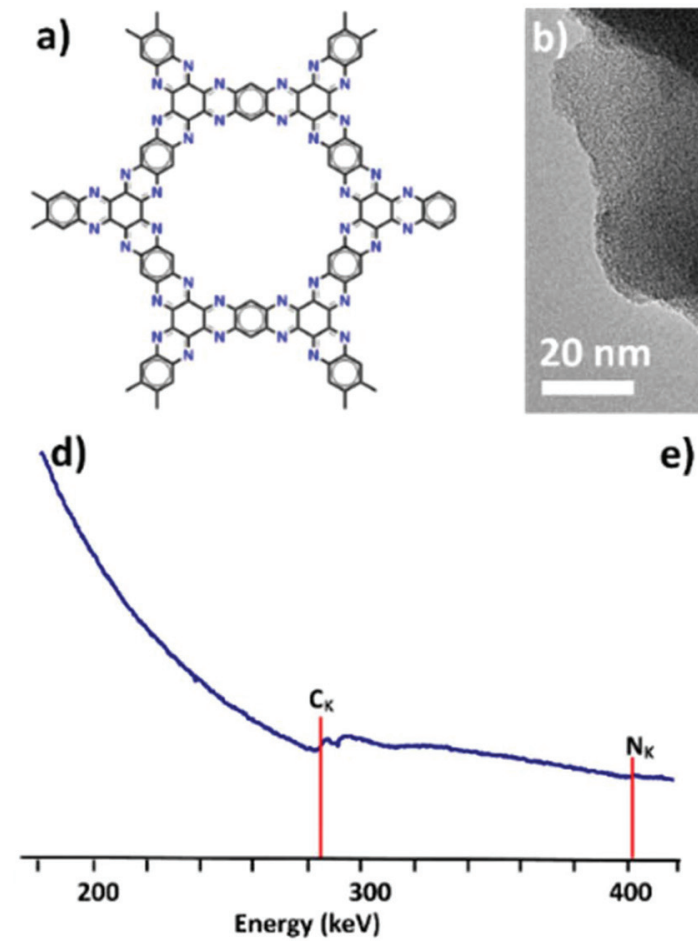
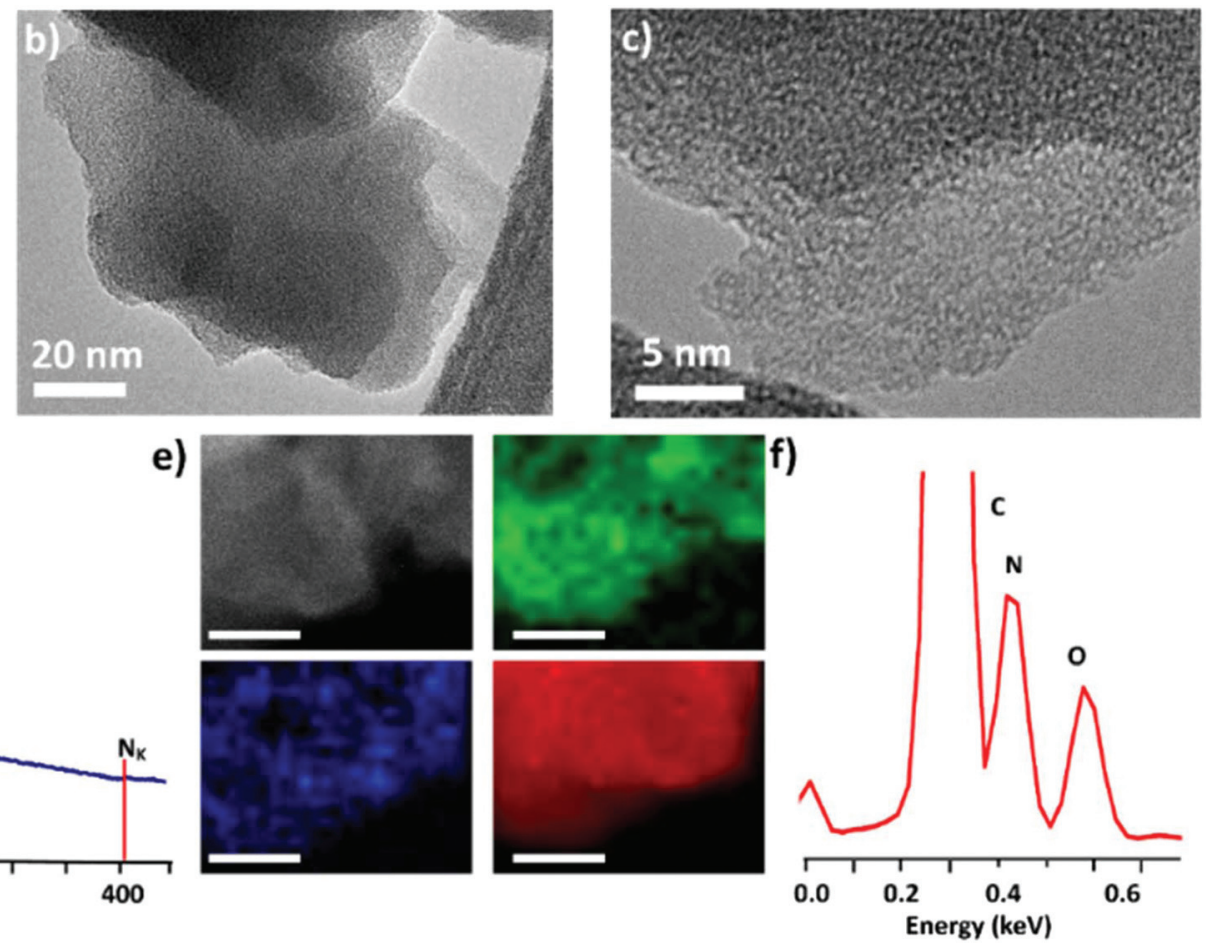

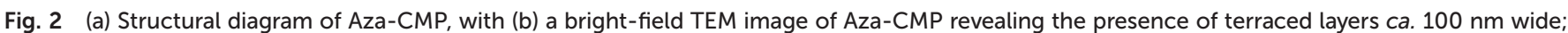

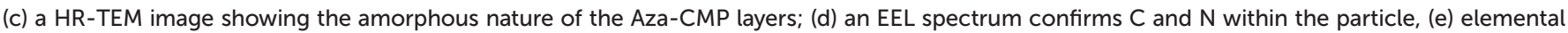

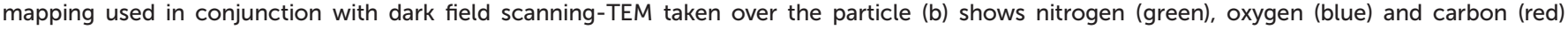
present; the scale bars are $10 \mathrm{~nm}$, and (f) EDX spectrum of Aza-CMP particle in (b) shows the occurrence of $\mathrm{C}$, $\mathrm{N}$ as well as $\mathrm{O}$ in the material. 


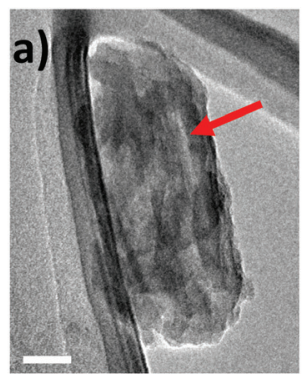

$-60^{\circ}$
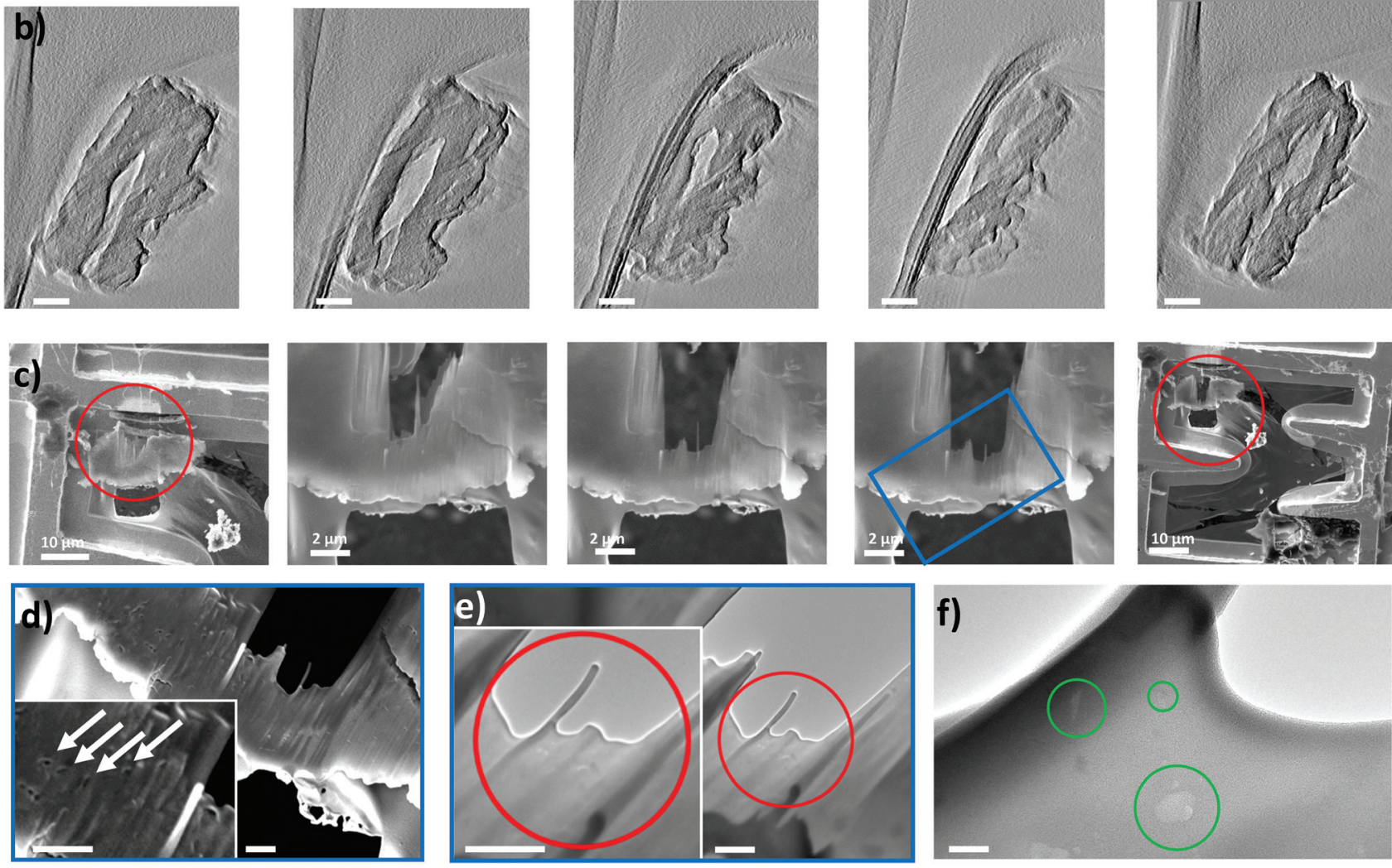

Fig. 3 (a) TEM tomographic tilt-series showing an Aza-CMP particle tilted through various angles allowing for a comprehensive visualization of the particle in three dimensions; the scale bars are $50 \mathrm{~nm}$ and red arrows point to an example of an internal mesopore. (b) Corresponding z-slices from the SIRT confirm the presence of mesopores running through the interior of the polymer particle; the scale bars are $50 \mathrm{~nm}$. (c) FIB etching of a particle of Aza-CMP reveals channels throughout the structure; the red circle (c, first panel) highlights the area of sample which was milled using the FIB, and the red circle (c, final panel) shows Aza-CMP post-milling and the blue rectangle in the fourth panel is the area of focus in (d), a higher resolution SEM image of the sample after FIB etching. Mesopores in the structure are revealed (d, inset, white arrows) and these are also shown in a TEM image of the same area (e) (the scale bars in (d) and (e) are $1 \mu \mathrm{m}$ ). The area within the red circle is magnified and shown in (f) where mesoporous cavities ca. $10-20 \mathrm{~nm}$ wide are visible. The scale bar is $20 \mathrm{~nm}$.

$\mathrm{Ga}^{+}$ions with a current of $0.5 \mathrm{nA}$ able to gradually etch the organic material (Fig. 3c series) and thus revealing pores $c a$. 20-50 nm. After the FIB etching the Aza-CMP particle was further analysed using a field emission gun SEM (Fig. 3d) capable of higher image resolution (Fig. 3e). Importantly, the FIB-SEM results are in good agreement with the TEM tomography, and a post-etched HR-TEM image (Fig. 3f), demonstrating the surprising mesopores penetrating the Aza-CMP, running deep inside the particle, similar to holes in a Swiss cheese, which are not expected from the molecular structure of this Aza-CMP material.
The unexpected extrinsic mesoporosity of Aza-CMP discovered by nanoscale analysis manifests itself in adsorption-desorption isotherms of $\mathrm{N}_{2}$ and $\mathrm{CO}_{2}$ after activation of the solid CMP under dynamic vacuum $\left(10^{-3} \mathrm{mbar}\right)$ at $100{ }^{\circ} \mathrm{C}$ overnight. The $\mathrm{N}_{2}$ isotherm features a hysteresis loop above $P / P_{0}=0.5$ which is characteristic of a mesoporous material (Fig. 4a). Gas uptake is low but measurable, which is likely due to diffusion limitations for a BET value of $23.8 \mathrm{~m}^{2} \mathrm{~g}^{-1}$. This value is much smaller than that displayed for related Aza-CMPs prepared by ionothermal methods, ${ }^{25}$ but consistent with the expected porosity for this material when synthesised by a solvothermal reac- 

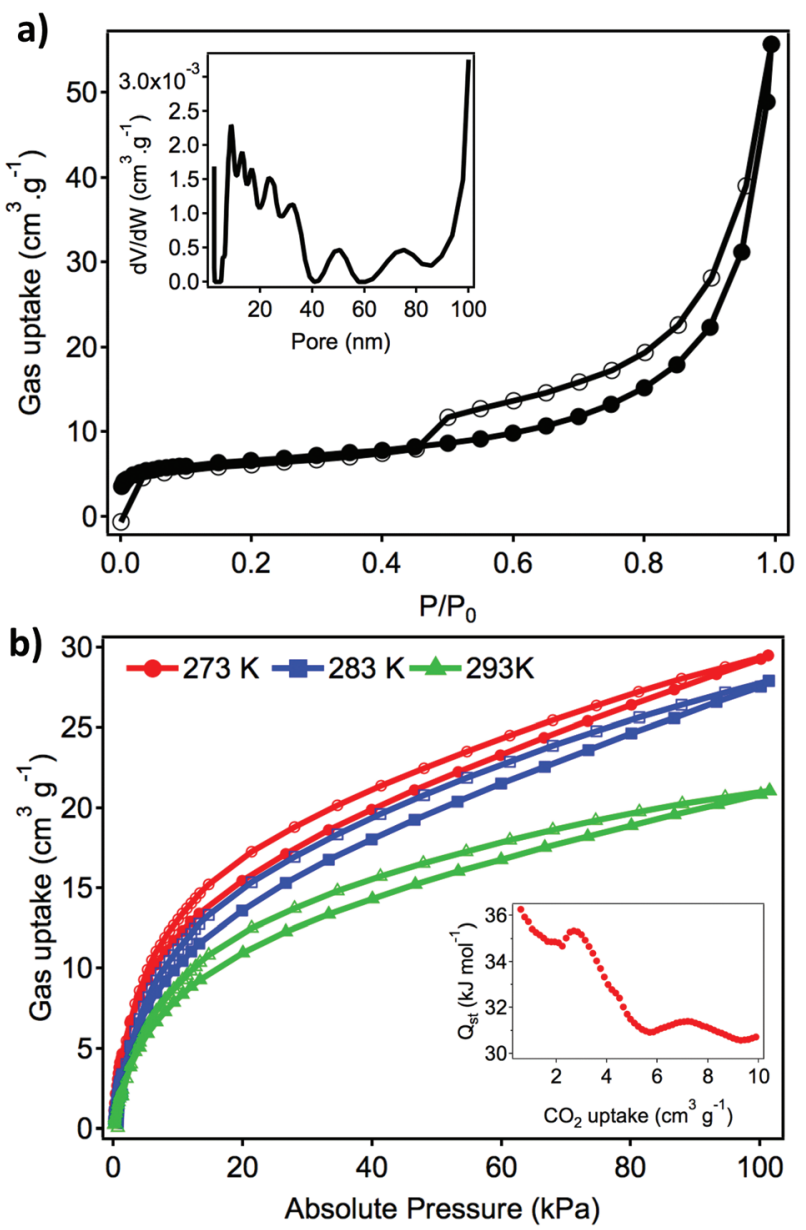

Fig. 4 (a) $\mathrm{N}_{2}$ Adsorption (black points)-desorption (white points) isotherm of Aza-CMP. The pore size distribution (inset) confirms the mesoporous nature of the defects; (b) the $\mathrm{CO}_{2}$ gas sorption isotherms at $273 \mathrm{~K}, 283 \mathrm{~K}$ and $293 \mathrm{~K}$, the filled points representing adsorption and the clear points representing desorption, with the calculated isosteric heat of adsorption $\left(Q_{s t}\right.$ inset).

tion. ${ }^{23}$ Analysis of the pore size distribution by applying a nonlinear solid density functional theory is in excellent agreement with the disordered nature of Aza-CMP particles, with a broad distribution of mesopore diameters centred between $5-40 \mathrm{~nm}$ (Fig. $4 \mathrm{a}$ inset). ${ }^{29}$ In turn, $\mathrm{CO}_{2}$ sorption is significantly higher; Aza-CMP adsorbs from 5.8 to 4.1 weight percent of $\mathrm{CO}_{2}$ at 1 bar between 273 and $293 \mathrm{~K}$ with an isosteric heat adsorption of $36.0 \mathrm{~kJ} \mathrm{~mol}^{-1}$ (Fig. 4b). This value decreases with the coverage and suggests a strong interaction between $\mathrm{CO}_{2}$ molecules and the framework due to the incorporation of nitrogen atoms into the pore walls. ${ }^{30}$

In order to elucidate whether the observed internal mesopores are formed during the Aza-CMP synthesis or are an artefact of post-synthetic handing, different post-synthesis treatment procedures were tested, such as sonication in different organic solvents, and dry exfoliation followed by deposition, using mechanical grinding (ESI Fig. S5†). Similarly TEM analysis was performed at a selection of different accelerating vol- tages and dose rates in order to reveal whether electron beam damage played a part in the defect formation and it was found that there were no variations to the structure of the particles when using the different procedures (ESI Fig. S6 $†$ ). Instead, we found that the physical size and morphology of the Aza-CMP material remained very similar regardless of the treatment and therefore, the mesopores are most likely to emerge during the Aza-CMP synthesis as a result of the incomplete polycondensation of the hexaketocyclohexane and tetra-amine which is consistent with the abundance of oxygen as observed in the EEL and EDX spectra (Fig. 2d-f). The mesopores may form as a result of the reactants assembling around the solvent water prior to condensation, driven by the affinity of the hexaketocyclohexane to form hydrogen bonds with the solvent water molecules. Once the layered porous Aza-CMP particle has formed the water evaporates leaving internal voids which we observe as mesopores during the analysis. This process is akin to the 'ball on a freezing pond' model where if a ball (a cluster of solvent molecules) is placed on water (reactants giving rise to the CMP, Fig. 5a) which subsequently freezes (Fig. 5b), removing the ball results in a hole giving the presence of pores within a structure (Fig. 5c) which are defined by the dynamics of the polymerisation rather than the intrinsic structure of Aza-CMP polymer itself. A similar mechanism would explain the mesoporosity of the COF-5 particles, with the only differ-


Fig. 5 A model showing how the mesopores may arise utilising the 'ball in a freezing pond' model. A ball is placed on water (a) which subsequently freezes (b) removing the ball results in a hole giving the presence of pores within a solid structure (c); for a complete polycondensation (d), no $\mathrm{O}$ atoms should remain. However, the removal of building blocks of the Aza-CMP network (schematically shown, e) results in the formation of pores terminated by ketone groups (represented by the blue hexagons). 
ence being that the solid domains in the COF-5 are highly crystalline due to a more reversible nature of the COF-5 polycondensation than the CMP. In addition to the trapped solvent molecules, the defects can be produced by the encapsulation of molecular precursors, intermediates and soluble small molecular weight polymeric layers that were washed during the Soxhlet extraction.

Our hypothesis also explains the presence of the oxygen observed in the elemental analysis, as incomplete condensation has occurred due to the steric interference from the solvent cluster and hence the edges of the defects will be terminated with carbonyl groups of incompletely reacted hexaketocyclohexane. A quantitative EDX analysis can be utilised to estimate the atomic percentage of particular elements within nanomaterials, ${ }^{31}$ which can give a reasonable estimation to the amount of ketone groups remaining within the CMP particle (ESI $\dagger$ ). Therefore, the atomic percent of oxygen can be used as a measure of completeness of polycondensation, which is expected to be zero for a perfect Aza-CMP (Fig. 5d), but in our case it is 7 atomic\% indicating that approximately $27 \%$ of the ketone groups remain unreacted. Due to the topology of the CMP defects the remaining ketone groups are expected to be lining the surface of the mesopores (Fig. 5e) thus determining their chemical properties.

\section{Conclusions}

Aperiodic mesopores have been discovered in both the crystalline COF-5 and non-crystalline Aza-CMP materials using a combination of high-resolution TEM, tomography and image reconstruction as a powerful analytical approach for the structural characterisation of complex disordered organic particles. The nanoscale structure of COF-5 is susceptible to electron beam damage, and care should be taken when analysing COFs by electron microscopy to avoid imaging artefacts. In contrast, Aza-CMP is highly stable which allowed a full 3D analysis by FIB-SEM followed by TEM imaging of cross sections of this material, thus providing a complete three-dimensional understanding of Aza-CMP, and also shedding light on the mechanisms of its formation. The mesoporous structures penetrating through the Aza-CMP and COF-5 particles are surprising and could be fully analysed and understood only by an innovative combination of several electron microscopy methods which we have developed in this study. The Aza-CMP mesopores appear to be highly stable, and remain intact during various treatments (e.g. solvent extraction, ultrasound, FIB etching, or heating in vacuum), akin to inorganic porous structures. This discovery opens a wealth of opportunities for future applications of CMPs in the areas of extraction, catalysis, gas storage or fuel-cells all of which require durable mesoporous materials. On the methodological level, our integrated analytical approach can now be applied to all types of CMP and COF materials (and other disordered molecular materials deemed to be too complex or even 'structureless' in the past) to enable meaningful structural characterisation at the nanoscale, pushing the frontier of chemical knowledge beyond the traditional types of crystalline molecular solids, and discovering nanoscale structural features inaccessible by other means of analysis.

\section{Experimental}

\section{Gas sorption measurements}

Gas sorption measurements were recorded using Micromeritics3Flex apparatus at relative pressures up to 1 bar and performed ex situ on activated solids. Samples were degassed overnight at $100{ }^{\circ} \mathrm{C}$ and $10^{-6}$ Torr prior to analysis. Nitrogen adsorption-desorption isotherms were recorded at $77 \mathrm{~K}$ and the pore size distribution was analysed by using a solid density functional theory (NLDFT) for the adsorption branch by assuming a cylindrical pore model. $\mathrm{CO}_{2}$ adsorption was collected at $273 \mathrm{~K}, 283 \mathrm{~K}$ and $293 \mathrm{~K}$ using a Micromeritics' ISO Controller to keep the temperature constant. The isosteric heat of adsorption of $\mathrm{CO}_{2}$ was determined by using the Clausius-Clapeyron equation as implemented in the MicroActive Analysis software.

\section{Electron microscopy}

All samples were prepared by either dispersion in propan-2-ol or acetone using an ultrasonic bath and drop cast onto lacey carbon-coated copper TEM grids (Agar), or by dry deposition involving gentle grinding with a pestle and mortar. TEM imaging was carried out on a either a JEOL 2100 FEG-TEM microscope operated at $100 \mathrm{kV}$ or $200 \mathrm{kV}$, or a JEOL $2100+$ TEM operated at either $80 \mathrm{kV}$ or $200 \mathrm{kV}$. Tilt series were taken using a Gatan 916 high tilt tomography holder, acquiring images at $1^{\circ}$ tilt intervals.

\section{EDX spectroscopy}

Local EDX spectra were acquired for samples mounted on TEM grids using an Oxford Instruments X-Max 80 detector and INCA X-ray microanalysis software. The electron beam was condensed onto areas of specimen suspended over holes of the amorphous carbon film.

\section{Scanning electron microscopy}

High resolution SEM was performed on a JEOL $7100 \mathrm{~F}$ operated at $5 \mathrm{kV}$. FIB-SEM was carried out using an FEI Quanta200 3D Dual Beam FIB-SEM operated at $10 \mathrm{kV}$. A beam of $\mathrm{Ga}^{+}$ions, accelerated at $30 \mathrm{kV}$ and with a current $0.5 \mathrm{nA}$ was used for the etching procedure.

\section{Simultaneous iterative reconstruction technique}

Tomographic reconstruction was performed via the IMOD v4.9.3 software, using 100 iterations of the SIRT algorithm.

\section{Conflicts of interest}

There are no conflicts to declare. 


\section{Acknowledgements}

We acknowledge the support of the Engineering and Physical Science Research Council (EPSRC) for funding, and the Nanoscale and Microscale Research Centre (nmRC) for access to instrumentation. We are also grateful to the Basque Science Foundation for Science (Ikerbasque), POLYMAT, the University of the Basque Country (Grupo de Investigación GIU17/054 and SGIker), Gobierno de España (Ministerio de Economía y Competitividad CTQ201677970-R, CTQ2017-83486-P and FPU16/04162), Gobierno Vasco (BERC program). This project has received funding from the European Union's Horizon 2020 research and innovation programme under grant agreement no 664878. This project has received funding from the European Research Council (ERC) under the European Union's Horizon 2020 research and innovation programme (grant agreements 722951 and 714122).

\section{References}

1 C. L. Tan, X. H. Cao, X. J. Wu, Q. Y. He, J. Yang, X. Zhang, J. Z. Chen, W. Zhao, S. K. Han, G. H. Nam, M. Sindoro and H. Zhang, Chem. Rev., 2017, 117, 6225-6331.

2 B. M. Savoie, K. L. Kohlstedt, N. E. Jackson, L. X. Chen, M. O. de la Cruz, G. C. Schatz, T. J. Marks and M. A. Ratner, Proc. Natl. Acad. Sci. U. S. A., 2014, 111, 10055-10060.

3 N. Huang, P. Wang and D. L. Jiang, Nat. Rev. Mater., 2016, 1, 16068.

4 Y. H. Xu, S. B. Jin, H. Xu, A. Nagai and D. L. Jiang, Chem. Soc. Rev., 2013, 42, 8012-8031.

5 Y. Pramudya and J. L. Mendoza-Cortes, J. Am. Chem. Soc., 2016, 138, 15204-15213.

6 L. Guo, X. Zeng and D. Cao, Sens. Actuators, B, 2016, 226, 273-278.

7 P. Wang, M. Kang, S. Sun, Q. Liu, Z. Zhang and S. Fang, Chin. J. Chem., 2014, 32, 838-843.

8 V. S. Vyas, M. Vishwakarma, I. Moudrakovski, F. Haase, G. Savasci, C. Ochsenfeld, J. P. Spatz and B. V. Lotsch, Adv. Mater., 2016, 28, 8749-8754.

9 L. Bai, S. Z. F. Phua, W. Q. Lim, A. Jana, Z. Luo, H. P. Tham, L. Zhao, Q. Gao and Y. Zhao, Chem. Commun., 2016, 52, 4128-4131.

10 S. Ghasimi, S. A. Bretschneider, W. Huang, K. Landfester and K. A. I. Zhang, Adv. Sci., 2017, 4, 1700171.

11 Y. B. Zhou, Y. Q. Wang, L. C. Ning, Z. C. Ding, W. L. Wang, C. K. Ding, R. H. Li, J. J. Chen, X. Lu, Y. J. Ding and Z. P. Zhan, J. Am. Chem. Soc., 2017, 139, 3966-3969.

12 R. Gutzler and D. F. Perepichka, J. Am. Chem. Soc., 2013, 135, 16585-16594.

13 P. Zhu and V. Meunier, J. Chem. Phys., 2012, 137, 244703.

14 R. P. Bisbey and W. R. Dichtel, ACS Cent. Sci., 2017, 3, 533-543.
15 Y. Liu, Y. Ma, Y. Zhao, P. Oleynikov, O. Terasaki and O. Yaghi, in European Microscopy Congress 2016: Proceedings, Wiley-VCH Verlag GmbH \& Co. KGaA, 2016, DOI: 10.1002/9783527808465.EMC2016.6799.

16 Y.-B. Zhang, J. Su, H. Furukawa, Y. Yun, F. Gándara, A. Duong, X. Zou and O. M. Yaghi, J. Am. Chem. Soc., 2013, 135, 16336-16339.

17 F. Haase, K. Gottschling, L. Stegbauer, L. S. Germann, R. Gutzler, V. Duppel, V. S. Vyas, K. Kern, R. E. Dinnebier and B. V. Lotsch, Mater. Chem. Front., 2017, 1, 1354-1361.

18 V. S. Vyas, F. Haase, L. Stegbauer, G. Savasci, F. Podjaski, C. Ochsenfeld and B. V. Lotsch, Nat. Commun., 2015, 6, 8508.

19 Y. W. Peng, Y. Huang, Y. H. Zhu, B. Chen, L. Y. Wang, Z. C. Lai, Z. C. Zhang, M. T. Zhao, C. L. Tan, N. L. Yang, F. W. Shao, Y. Han and H. Zhang, J. Am. Chem. Soc., 2017, 139, 8698-8704.

20 D. D. Medina, J. M. Rotter, Y. H. Hu, M. Dogru, V. Werner, F. Auras, J. T. Markiewicz, P. Knochel and T. Bein, J. Am. Chem. Soc., 2015, 137, 1016-1019.

21 S. B. Kalidindi, H. Oh, M. Hirscher, D. Esken, C. Wiktor, S. Turner, G. Tendeloo and R. A. Fischer, Chem. - Eur. J., 2012, 18, 10848-10856.

22 A. P. Cote, A. I. Benin, N. W. Ockwig, M. O'Keeffe, A. J. Matzger and O. M. Yaghi, Science, 2005, 310, 11661170.

23 A. B. Marco, D. Cortizo-Lacalle, I. Perez-Miqueo, G. Valenti, A. Boni, J. Plas, K. Strutyński, S. De Feyter, F. Paolucci, M. Montes, A. N. Khlobystov, M. Melle-Franco and A. Mateo-Alonso, Angew. Chem., Int. Ed., 2017, 56, 69466951.

24 V. Briega-Martos, A. Ferre-Vilaplana, A. de la Peña, J. L. Segura, F. Zamora, J. M. Feliu and E. Herrero, ACS Catal., 2017, 7, 1015-1024.

25 Y. Kou, Y. H. Xu, Z. Q. Guo and D. L. Jiang, Angew. Chem., Int. Ed., 2011, 50, 8753-8757.

26 H. Lee, K. Kim, S. H. Kang, Y. Kwon, J. H. Kim, Y. K. Kwon, R. Ryoo and J. Y. Park, Sci. Rep., 2017, 7, 11460.

27 S. Los, L. Duclaux, W. Kempinski and M. Polomska, Microporous Mesoporous Mater., 2010, 130, 21-25.

28 R. J. Gaudette, D. H. Brooks, C. A. DiMarzio, M. E. Kilmer, E. L. Miller, T. Gaudette and D. A. Boas, Phys. Med. Biol., 2000, 45, 1051-1070.

29 P. I. Ravikovitch, G. L. Haller and A. V. Neimark, Adv. Colloid Interface Sci., 1998, 76, 203-226.

30 X. Wang, Y. Zhao, L. Wei, C. Zhang and J.-X. Jiang, J. Mater. Chem. A, 2015, 3, 21185-21193.

31 C. T. Stoppiello, J. Biskupek, Z. Y. Li, G. A. Rance, A. Botos, R. M. Fogarty, R. A. Bourne, J. Yuan, K. R. J. Lovelock, P. Thompson, M. W. Fay, U. Kaiser, T. W. Chamberlain and A. N. Khlobystov, Nanoscale, 2017, 9, 14385-14394. 